\title{
Class Number Formulae of Dirichlet Type
}

\author{
By Richard H. Hudson and Kenneth S. Williams*
}

\begin{abstract}
Applying a theorem of Johnson and Mitchell, some new class number formulae are derived.
\end{abstract}

Throughout this paper $h(D)$ denotes the class number of the quadratic field $Q(\sqrt{D})$ of discriminant $D$. In 1840 Dirichlet [4] proved (among others) the class number formulae

$$
h(-p)=\frac{1}{3} \sum_{0<n<p / 2}\left(\frac{n}{p}\right), \quad p \equiv 3(\bmod 8),
$$

and

$$
h(-p)=\sum_{0<n<p / 2}\left(\frac{n}{p}\right), \quad p \equiv 7(\bmod 8),
$$

where, here and throughout the paper, $p$ denotes a prime greater than 3 . Since then many other similar formulae have appeared in the literature; see for example [1], [3]-[16]. We just give two examples. In the work of Karpinski [14, p. 15] we find

$$
h(-5 p)=-4 \sum_{4 p / 10<n<5 p / 10}\left(\frac{n}{p}\right), \quad p \equiv 31,39(\bmod 40),
$$

while

$$
h(-3 p)=2 \sum_{0<n<p / 3}\left(\frac{n}{p}\right), \quad p \equiv 1(\bmod 4),
$$

appears in the paper of Lerch [15, p. 402]. In some research of the second author the following formula arose:

$$
h(-7 p)=-2 \sum_{5 p / 14<n<6 p / 14}\left(\frac{n}{p}\right), \quad p \equiv 5(\bmod 8) .
$$

This result does not seem to have been stated explicitly before. The authors decided therefore to determine all such class number formulae of the type given in (1)-(5) within certain limits.

Received January 15, 1982.

1980 Mathematics Subject Classification. Primary 12A50, 12-04; Seconary 12A25.

Key words and phrases. Dirichlet type class number formulae, class numbers of imaginary quadratic number fields.

* Research supported by Natural Sciences and Engineering Research Council of Canada Grant No. A-7233. 
All of these formulae are of the form

$$
h(-d p)=c \sum_{(k-1) p / l d<n<k p / l d}\left(\frac{n}{p}\right), \quad p \equiv r\left(\bmod 4 \prod_{q \mid l d} q\right),
$$

where

(i) $d$ is a positive integer such that $-d p$ is the discriminant of an imaginary quadratic field,

(ii) $l$ is a positive integer,

(iii) $k$ is a positive integer satisfying $1 \leqslant k \leqslant\left[\frac{1}{2}(l d+1)\right]$,

(iv) $q$ runs through primes dividing $l d$,

(v) $r$ is an integer satisfying $1 \leqslant r \leqslant 4 \prod_{q \mid l d} q,(r, 2 l d)=1$,

(vi) $c$ is a rational number.

We call a formula of the form (6) a class number formula of Dirichlet type. A computer search was designed to find all such formulae for which the parameters $d$ and $l$ satisfy $1 \leqslant d \leqslant 28,1 \leqslant l d \leqslant 30$. Within these limits our search revealed 127 formulae of Dirichlet type. (We note that it is not impossible for a data input error to have resulted in a missed formula.) Of these 127 formulae, which are summarized in the following table, 84 were found in the literature and 43 appear to be new. Following Table 1 we indicate how the new formulae are proved.

TABLE 1

Dirichlet type class number formulae

\begin{tabular}{|c|c|c|c|c|c|c|}
\hline$d$ & $l$ & $k$ & $c$ & $r$ & $4 \prod_{q \mid l d} q$ & References \\
\hline 1 & 2 & 1 & $1 / 3$ & 3 & 8 & Dirichlet [4] \\
\hline 1 & 2 & 1 & 1 & 7 & 8 & “ " \\
\hline 1 & 3 & 1 & $1 / 2$ & 7 & 12 & Lerch [15] \\
\hline 1 & 3 & 1 & 1 & 11 & 12 & “ " \\
\hline 1 & 4 & 1 & 1 & 7 & 8 & Dirichlet [4] \\
\hline 1 & 4 & 2 & $1 / 3$ & 3 & 8 & “ " " \\
\hline 1 & 6 & 1 & 1 & 7 & 24 & Holden [7] \\
\hline 1 & 6 & 1 & 1 & 11 & 24 & “ ” \\
\hline 1 & 6 & 1 & -1 & 19 & 24 & “ \\
\hline 1 & 6 & 1 & 1 & 23 & 24 & “ \\
\hline 1 & 6 & 2 & 1 & 7 & 24 & “ \\
\hline 1 & 6 & 2 & $1 / 3$ & 19 & 24 & “ \\
\hline 1 & 6 & 3 & -1 & 7 & 24 & “ \\
\hline 1 & 6 & 3 & $1 / 2$ & 11 & 24 & “ \\
\hline 1 & 6 & 3 & 1 & 19 & 24 & “ " \\
\hline 1 & 10 & 1 & 1 & 11 & 40 & Karpinski [14] \\
\hline 1 & 10 & 1 & 1 & 19 & 40 & . " " \\
\hline 1 & 12 & 3 & -1 & 11 & 24 & “ \\
\hline 1 & 12 & 3 & 1 & 19 & 24 & “ \\
\hline 1 & 12 & 4 & 1 & 7 & 24 & “ \\
\hline 1 & 12 & 4 & 1 & 11 & 24 & “ \\
\hline 1 & 12 & 4 & $1 / 2$ & 19 & 24 & \\
\hline 1 & 14 & 3 & -1 & 11 & 56 & \\
\hline 1 & 14 & 3 & -1 & 43 & 56 & \\
\hline 1 & 14 & 3 & -1 & 51 & 56 & \\
\hline 1 & 14 & 6 & 1 & 11 & 56 & \\
\hline 1 & 14 & 6 & 1 & 43 & 56 & \\
\hline
\end{tabular}


TABLE 1

Dirichlet type class number formulae

\begin{tabular}{|c|c|c|c|c|c|c|}
\hline$d$ & 1 & $k$ & $c$ & $r$ & $4 \prod_{q \mid l d} q$ & References \\
\hline 1 & 14 & 6 & 1 & 51 & 56 & Karpinski [14] \\
\hline 1 & 18 & 8 & -1 & 19 & 24 & " " \\
\hline 1 & 20 & 6 & 1 & 11 & 40 & \\
\hline 1 & 20 & 6 & 1 & 19 & 40 & \\
\hline 1 & 30 & 10 & 1 & 19 & 120 & \\
\hline 1 & 30 & 10 & $1 / 2$ & 43 & 120 & Johnson and Mitchell [13] \\
\hline 1 & 30 & 10 & $1 / 2$ & 67 & 120 & “" " \\
\hline 1 & 30 & 10 & 1 & 83 & 120 & \\
\hline 1 & 30 & 10 & 1 & 91 & 120 & \\
\hline 1 & 30 & 10 & 1 & 107 & 120 & \\
\hline 3 & 1 & 1 & 2 & 1 & 12 & Lerch [15] \\
\hline 3 & 1 & 1 & 2 & 5 & 12 & “ " \\
\hline 3 & 1 & 2 & -1 & 1 & 12 & “ $"$ \\
\hline 3 & 1 & 2 & -1 & 5 & 12 & “ $\quad$, \\
\hline 3 & 2 & 1 & 1 & 1 & 24 & “ " " \\
\hline 3 & 2 & 1 & 1 & 17 & 24 & “ $\quad "$ \\
\hline 3 & 2 & 2 & -2 & 1 & 24 & “ \\
\hline 3 & 2 & 2 & -2 & 17 & 24 & “ \\
\hline 3 & 2 & 2 & 2 & 5 & 24 & “ \\
\hline 3 & 2 & 2 & 2 & 13 & 24 & “ \\
\hline 3 & 2 & 3 & -2 & 1 & 24 & “ $\quad$, \\
\hline 3 & 2 & 3 & -2 & 5 & 24 & “ \\
\hline 3 & 2 & 3 & -2 & 13 & 24 & “ \\
\hline 3 & 2 & 3 & -2 & 17 & 24 & \\
\hline 3 & 4 & 1 & 2 & 17 & 24 & Karpinski [14] \\
\hline 3 & 4 & 2 & 2 & 17 & 24 & “ " \\
\hline 3 & 4 & 5 & -2 & 5 & 24 & “ \\
\hline 3 & 4 & 5 & -1 & 17 & 24 & “ " \\
\hline 3 & 4 & 6 & 2 & 17 & 24 & “ \\
\hline 3 & 10 & 7 & 2 & 41 & 120 & \\
\hline 3 & 10 & 7 & 2 & 89 & 120 & \\
\hline 4 & 1 & 1 & 2 & 1 & 8 & Dirichlet [4] \\
\hline 4 & 1 & 1 & 2 & 5 & 8 & “ " " \\
\hline 4 & 1 & 2 & -2 & 1 & 8 & “ \\
\hline 4 & 1 & 2 & -2 & 5 & 8 & “ $\quad "$ \\
\hline 4 & 3 & 1 & 2 & 13 & 24 & Karpinski [14] \\
\hline 4 & 3 & 2 & -2 & 13 & 24 & " " " \\
\hline 4 & 3 & 3 & 2 & 5 & 24 & “ \\
\hline 4 & 3 & 3 & 2 & 13 & 24 & “ \\
\hline 4 & 3 & 6 & -2 & 13 & 24 & “ \\
\hline 5 & 1 & 2 & 2 & 3 & 20 & Lerch [15] \\
\hline 5 & 1 & 2 & 2 & 7 & 20 & " " \\
\hline 5 & 1 & 2 & 2 & 11 & 20 & “ $"$ \\
\hline 5 & 1 & 2 & 2 & 19 & 20 & . “ \\
\hline 5 & 2 & 2 & -4 & 11 & 40 & Karpinski [14] \\
\hline 5 & 2 & 2 & -4 & 19 & 40 & " " \\
\hline 5 & 2 & 2 & 4 & 31 & 40 & “ $\quad "$ \\
\hline 5 & 2 & 2 & 4 & 39 & 40 & “ $"$ \\
\hline 5 & 2 & 3 & 4 & 11 & 40 & “ \\
\hline
\end{tabular}


TABLE 1

Dirichlet type class number formulae

\begin{tabular}{|c|c|c|c|c|c|c|}
\hline$d$ & $l$ & $k$ & $c$ & $r$ & $4 \prod_{q \mid l d} q$ & References \\
\hline 5 & 2 & 3 & 4 & 19 & 40 & Karpinski [14] \\
\hline 5 & 2 & 3 & $4 / 3$ & 31 & 40 & “ " \\
\hline 5 & 2 & 3 & $4 / 3$ & 39 & 40 & “ " \\
\hline 5 & 2 & 4 & 4 & 11 & 40 & “ " \\
\hline 5 & 2 & 4 & 4 & 19 & 40 & “ " \\
\hline 5 & 2 & 4 & -4 & 31 & 40 & “ ” \\
\hline 5 & 2 & 4 & -4 & 39 & 40 & “ " \\
\hline 5 & 2 & 5 & -4 & 31 & 40 & “ " \\
\hline 5 & 2 & 5 & -4 & 39 & 40 & “ " \\
\hline 5 & 3 & 6 & 4 & 11 & 60 & \\
\hline 5 & 3 & 6 & 4 & 59 & 60 & \\
\hline 5 & 4 & 5 & 4 & 31 & 40 & \\
\hline 5 & 4 & 5 & 4 & 39 & 40 & \\
\hline 5 & 4 & 6 & 2 & 7 & 40 & \\
\hline 5 & 4 & 6 & 2 & 23 & 40 & \\
\hline 5 & 4 & 6 & 2 & 31 & 40 & \\
\hline 5 & 4 & 6 & 2 & 39 & 40 & \\
\hline 5 & 6 & 6 & -4 & 11 & 120 & \\
\hline 5 & 6 & 6 & -4 & 31 & 120 & \\
\hline 5 & 6 & 6 & -4 & 59 & 120 & \\
\hline 5 & 6 & 6 & -4 & 71 & 120 & \\
\hline 5 & 6 & 6 & -4 & 79 & 120 & \\
\hline 5 & 6 & 6 & -4 & 119 & 120 & \\
\hline 5 & 6 & 10 & -2 & 23 & 120 & \\
\hline 5 & 6 & 10 & -2 & 47 & 120 & \\
\hline 5 & 6 & 10 & -2 & 71 & 120 & \\
\hline 5 & 6 & 10 & -2 & 119 & 120 & \\
\hline 7 & 2 & 6 & -2 & 5 & 56 & \\
\hline 7 & 2 & 6 & -2 & 13 & 56 & \\
\hline 7 & 2 & 6 & -2 & 29 & 56 & \\
\hline 7 & 2 & 6 & -2 & 37 & 56 & \\
\hline 7 & 2 & 6 & -2 & 45 & 56 & \\
\hline 7 & 2 & 6 & -2 & 53 & 56 & \\
\hline 8 & 1 & 2 & 4 & 7 & 8 & Dirichlet [4] \\
\hline 8 & 1 & 3 & 4 & 7 & 8 & “ " " \\
\hline 8 & 1 & 4 & -4 & 7 & 8 & “ $"$ \\
\hline 8 & 3 & 4 & 4 & 7 & 24 & Karpinski [14] \\
\hline 8 & 3 & 4 & 4 & 23 & 24 & " " \\
\hline 8 & 3 & 9 & 4 & 24 & 24 & “ " \\
\hline 12 & 1 & 2 & 4 & 7 & 24 & “ " \\
\hline 12 & 1 & 2 & 4 & 11 & 24 & “ " \\
\hline 12 & 1 & 2 & 4 & 23 & 24 & “ " \\
\hline 12 & 1 & 5 & 4 & 11 & 24. & ، " \\
\hline 12 & 1 & 5 & 4 & 19 & 24 & “ " \\
\hline 12 & 1 & 5 & 4 & 23 & 24 & “ \\
\hline 12 & 1 & 6 & -4 & 7 & 24 & \\
\hline 12 & 1 & 6 & -4 & 23 & 24 & \\
\hline
\end{tabular}


TABLE 1

Dirichlet type class number formulae

\begin{tabular}{ccccccc}
\hline$d$ & $l$ & $k$ & $c$ & $r$ & $4 \prod_{q \mid l d} q$ & References \\
\hline 15 & 2 & 12 & -4 & 13 & 120 & Karpinski [14] \\
15 & 2 & 12 & -4 & 37 & 120 & “ ” \\
15 & 2 & 12 & -4 & 61 & 120 & \\
15 & 2 & 12 & -4 & 109 & 120 & \\
\hline
\end{tabular}

Henceforth we let $s(l d, k)$ denote the sum

$$
\sum_{(k-1) p / l d<n<k p / l d}\left(\frac{n}{p}\right) .
$$

In order to prove the $\mathbf{4 3}$ formulae in Table 1 for which we are unable to find a reference, it was necessary to complete the tables in [13] and to derive a new one (see Table 2) for $l d=30$ using the theorem of Johnson and Mitchell [13, pp. 117-118]. The 16 cases in Table 2 (listed as congruence classes modulo 120) arise from the 16 possible choices of sign for $(-1 / p),(2 / p),(3 / p),(5 / p)$. Since, in each case, exactly eleven of the equations in the theorem of Johnson and Mitchell are linearly independent, the 15 variables $s(30,1), \ldots, s(30,15)$ are given in terms of four parameters $A, B, C, D$. When 0 appears in Table 2 it denotes the number "zero" and not the letter "oh".

We just give the proofs of two of the class number formulae in Table 1 as the others are proved similarly using Table 2 and easily completed tables of Johnson and Mitchell. In particular, we prove

$$
h(-7 p)=-2 s(14,6), \quad p \equiv 5(\bmod 8),
$$

and

$$
h(-15 p)=-4 s(30,12), \quad p \equiv 13(\bmod 24) .
$$

To prove (7) and (8) we require the following result which is given in [2, Lemma 1]: if $p \equiv 1(\bmod 4)$ is prime and $d \neq 0(\bmod p)$ is the discriminant of an imaginary quadratic field, then

$$
h(d p)=\sum_{k=1}^{|d|}\left(\sum_{j=k}^{|d|}\left(\frac{d}{j}\right)\right) s(|d|, k) .
$$

Taking $d=-7$ in (9), we obtain

$$
h(-7 p)=-s(7,2)-2 s(7,3)-s(7,4)-2 s(7,5)-s(7,6) .
$$

As $s(7, r)=s(7,8-r)(r=1,2, \ldots, 7)$ for $p \equiv 1(\bmod 4)$, we have

$$
h(-7 p)=-2 s(7,2)-4 s(7,3)-s(7,4) \text {. }
$$

Next, as

$$
s(7, r)=s(14,2 r-1)+s(14,2 r) \quad(r=1,2, \ldots, 7)
$$




\begin{tabular}{|c|c|c|c|}
\hline $\begin{array}{c}n \\
\tilde{n} \\
\end{array}$ & 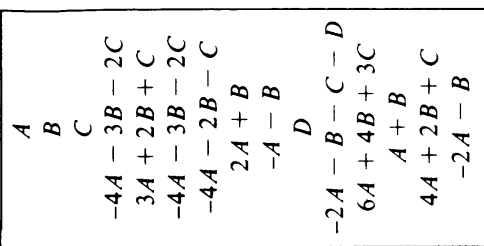 & 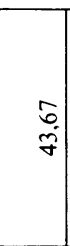 & 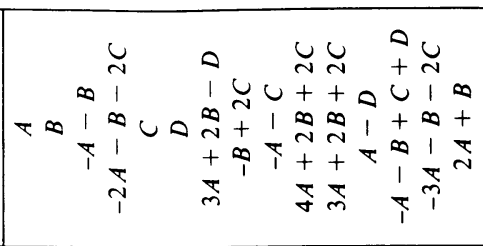 \\
\hline$\frac{\overline{0}}{\mathrm{i}}$ & 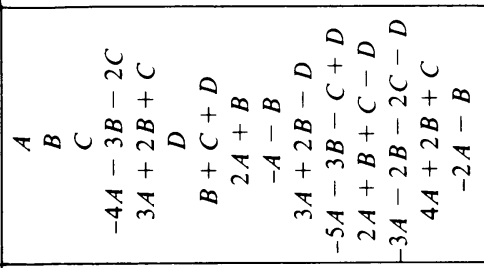 & $\begin{array}{l}\bar{\sigma} \\
\sigma\end{array}$ & 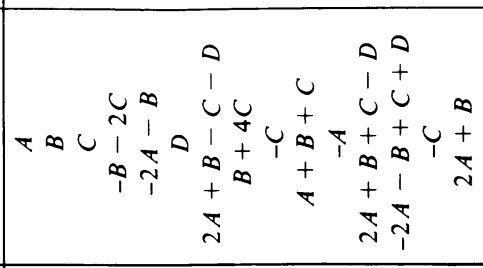 \\
\hline$\stackrel{\tilde{m}}{m}$ & 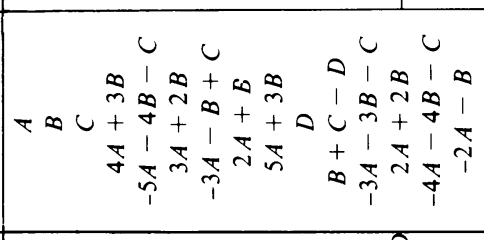 & & 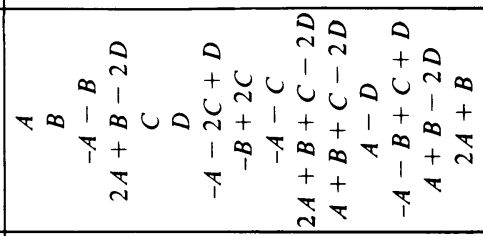 \\
\hline$\stackrel{m}{=}$ & 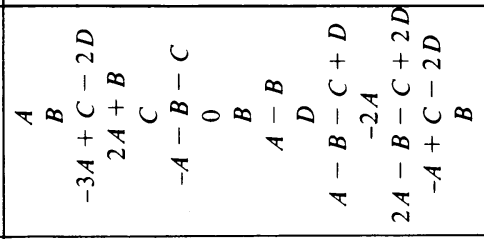 & $\frac{\dddot{0}}{\circ}$ & 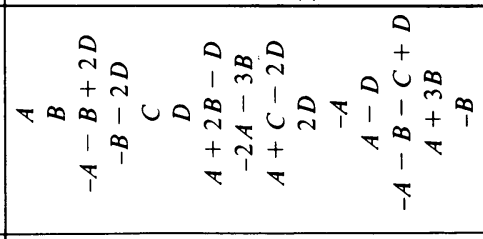 \\
\hline$\frac{8}{\circ}$ & 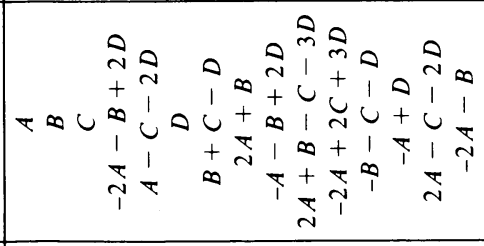 & $\stackrel{?}{=}$ & 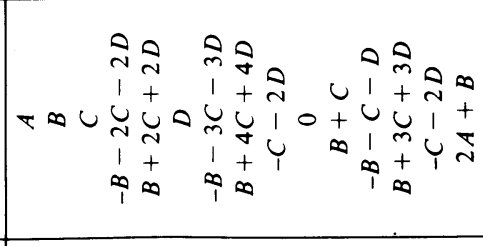 \\
\hline $\begin{array}{l}\hat{\alpha} \\
\vec{\sigma}\end{array}$ & 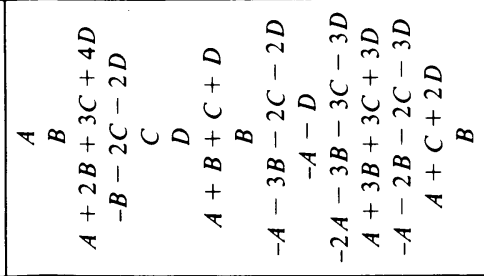 & $\frac{r}{m}$ & 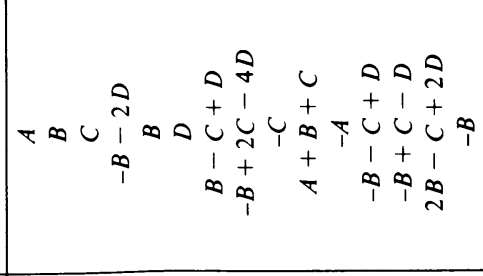 \\
\hline$\hat{\sigma}$ & 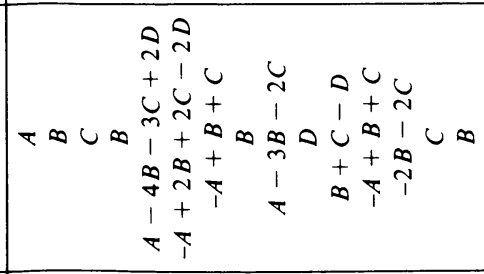 & $\underset{\tilde{v}}{\tilde{y}}$ & 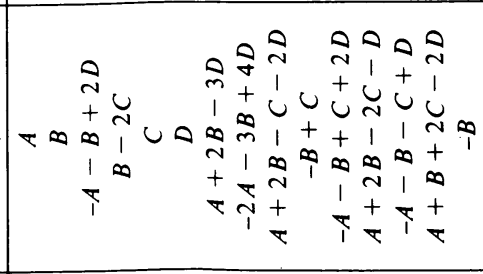 \\
\hline 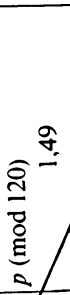 & 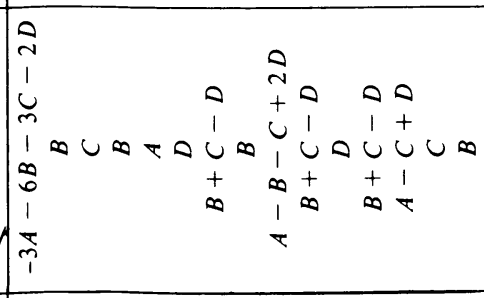 & 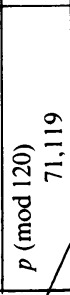 & 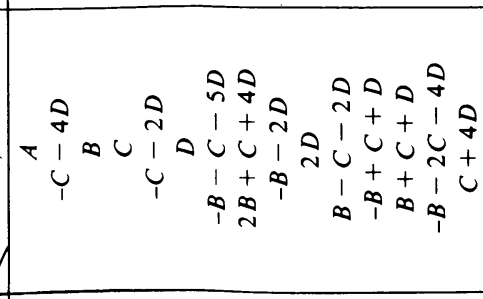 \\
\hline î & 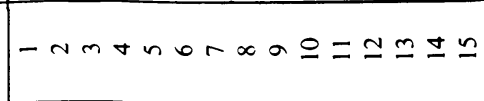 & 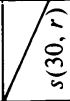 & $-N m+\backsim \circ r \infty a O= \pm m$ \\
\hline
\end{tabular}


and $s(14, r)=s(14,15-r)(r=1,2, \ldots, 14)$, we obtain

(10) $h(-7 p)=-2 s(14,3)-2 s(14,4)-4 s(14,5)-4 s(14,6)-2 s(14,7)$.

From the theorem of Johnson and Mitchell [13] (see the table on p. 122) we have, for $p \equiv 5,13,29,37,45,53(\bmod 56), s(14,1)=A, s(14,2)=B, s(14,3)=C, s(14,4)=$ $2 A+B, s(14,5)=A+B, s(14,6)=-2 A-2 B-C, s(14,7)=-2 A-B$. Using this in $(10)$, we obtain for $p \equiv 5(\bmod 8)$

$$
h(-7 p)=4 A+4 B+2 C=-2 s(14,6) \text {, }
$$

proving (7).

Next, taking $d=-15$ in (9), we obtain

$$
\begin{aligned}
h(-15 p)= & -s(15,2)-2 s(15,3)-2 s(15,4)-3 s(15,5) \\
& -3 s(15,6)-3 s(15,7)-2 s(15,8) \\
& -3 s(15,9)-3 s(15,10)-3 s(15,11) \\
& -2 s(15,12)-2 s(15,13)-s(15,14) .
\end{aligned}
$$

As $s(15, r)=s(15,16-r)(r=1,2, \ldots, 15)$ for $p \equiv 1(\bmod 4)$, we have

$$
\begin{aligned}
h(-15 p)= & -2 s(15,2)-4 s(15,3)-4 s(15,14)-6 s(15,5) \\
& -6 s(15,6)-6 s(15,7)-2 s(15,8) .
\end{aligned}
$$

Next, as $s(15, r)=s(30,2 r-1)+s(30,2 r) \quad(r=1,2, \ldots, 15)$ and $s(30, r)=$ $s(30,31-r)(r=1,2, \ldots, 30)$, we obtain

$$
\begin{aligned}
h(-15 p)= & -2 s(30,3)-2 s(30,4)-4 s(30,5)-4 s(30,6) \\
& -4 s(30,7)-4 s(30,8)-6 s(30,9)-6 s(30,10) \\
& -6 s(30,11)-6 s(30,12)-6 s(30,13)-6 s(30,14)-4 s(30,15) .
\end{aligned}
$$

Appealing to Table 2 we have, after simplifying the above, for $p \equiv 13,37$ $(\bmod 120)$,

$$
h(-15 p)=12 A+12 B+4 C=-4 s(30,12),
$$

and, for $p \equiv 61,109(\bmod 120)$,

$$
h(-15 p) \equiv 4 B+4 C+4 D=-4 s(30,12),
$$

completing the proof of (8).

Department of Mathematics and Statistics

University of South Carolina

Columbia, South Carolina 29208

Department of Mathematics and Statistics

Carleton University

Ottawa, Ontario K1S 5B6, Canada

1. Bruce C. Berndt, "Classical theorems on quadratic residues," Enseign. Math., v. 22, 1976, pp. 261-304.

2. James D. CURrie \& Kenneth S. Williams, "Class numbers and biquadratic reciprocity." (Submitted.)

3. G. L. DiRIChLET, "Recherches sur diverses applications de l'analyse infinitésimale à la théorie des nombres," J. Reine Angew. Math., v. 19, 1839, pp. 324-369.

4. G. L. DiRIChlet, "Recherches sur diverses applications de l'analyse infinitésimale à la théorie des nombres, second partie,” J. Reine Angew. Math., v. 21, 1840, pp. 134-155. 
5. J. W. L. Glaisher, "On the expression for the number of classes of a negative determinant, and on the numbers of positives in the octants of $P$," Quart. J. Math., v. 34, 1903, pp. 178-204.

6. H. Holden, "On various expressions for $h$, the number of properly primitive classes for a determinant $-p$, where $p$ is a prime of the form $4 n+3$ (first paper)," Messenger Math., v. 35, 1905/1906, pp. 73-80.

7. H. Holden, "On various expressions for $h$, the number of properly primitive classes for a determinant $-p$, where $p$ is of the form $4 n+3$, and is a prime or the product of different primes (second paper)," Messenger Math., v. 35, 1905/1906, pp. 102-110.

8. H. HoldEN, "On various expressions for $h$, the number of properly primitive classes for any negative determinant, not involving a square factor (third paper)," Messenger Math., v. 35, 1905/1906, pp. 110-117.

9. H. HoldEN, “On various expressions for $h$, the number of properly primitive classes for a negative determinant (fourth paper)," Messenger Math., v. 36, 1906/1907, pp. 69-75.

10. H. Holden, "On various expressions for $h$, the number of properly primitive classes for a determinant $-p$, where $p$ is of the form $4 n+3$, and is a prime or the product of different primes (addition to the second paper)," Messenger Math., v. 36, 1906/1907, pp. 75-77.

11. H. HoLDEN, "On various expressions for $h$, the number of properly primitive classes for a negative determinant not containing a square factor (fifth paper)," Messenger Math., v. 36, 1906/1907, pp. $126-134$.

12. H. HoldEN, "On various expressions for $h$, the number of properly primitive classes for any negative determinant, not containing a square factor (sixth paper)," Messenger Math., v. 37, 1907/1908, pp. $13-16$.

13. Wells Johnson \& Kevin J. Mitchell, "Symmetries for sums of the Legendre symbol," Pacific. J. Math., v. 69, 1977, pp. 117-124.

14. Louis C. KARPINSKI, “Über die Verteilung der quadratischen Reste," J. Reine Angew. Math., v. 127, 1904, pp. 1-19.

15. M. LeRCH, "Essais sur le calcul du nombre des classes de formes quadratiques binaires aux coefficients entiers," Acta Math., v. 29, 1905, pp. 333-424.

16. M. LERCH, "Essais sur le calcul du nombre des classes de formes quadratiques binaires aux coefficients entiers," Acta Math., v. 30, 1906, pp. 203-293. 